\title{
Endoplasmic-reticulum stress pathway-associated mechanisms of action of proteasome inhibitors in multiple myeloma
}

\author{
Masaki Ri ${ }^{1}$
}

Received: 5 April 2016 / Revised: 30 April 2016 / Accepted: 2 May 2016 / Published online: 12 May 2016

(C) The Japanese Society of Hematology 2016

\begin{abstract}
Bortezomib (BTZ), a proteasome inhibitor, was initially reported as an inhibitor of the NF-кB pathway, which plays a critical role in the pathogenesis of multiple myeloma (MM). The NF- $\kappa \mathrm{B}$ activity of MM cells is mediated via two distinct pathways, canonical and non-canonical, which show opposing activity after BTZ treatment in MM cells. Recent studies of proteasome inhibition in MM cells reveal that the accumulation of unfolded proteins in the endoplasmic reticulum (ER), referred to as ER stress, triggered the activity of several pro-apoptotic factors and sources of cell stress, such as the accumulation of reactive oxygen species (ROS), which is considered to be the main mechanism of action of BTZ-induced apoptosis. Several factors associated with ER stress and unfolded protein response (UPR) have been identified with the sensitivity of BTZ treatment. Low levels of XBP1, ATF3, and ATF4, which regulate UPR and ER stress-induced apoptosis, have been observed in poor responders to BTZ treatment, and three other genes, KLF9, Nampt, and CDK5, are associated with response to BTZ-containing therapy. These findings contribute to a better understanding of the mechanisms underlying BTZ-induced apoptosis in MM cells; however, further study is needed to develop potential predictive biomarkers of efficacy of BTZ-containing therapy.
\end{abstract}

Keywords Bortezomib · Multiple myeloma • Mechanism of action $\cdot$ ER stress $\cdot$ NF- $\kappa$ B

Masaki Ri

rrmasaki@ybb.ne.jp

1 Department of Hematology and Oncology, Nagoya City University Graduate School of Medical Sciences, 1 Kawasumi, Mizuho-chou, Mizuho-ku, Nagoya, Aichi 467-8601, Japan

\section{Introduction}

After the approval of proteasome inhibitor, bortezomib (BTZ), in the therapy of multiple myeloma (MM) in Japan, profound response, such as complete response (CR) or very good partial response (VGPR), has been often observed [1]. Although several adverse events, such as peripheral neuropathy and severe constipation, occurred during the treatment, BTZ is now widely used in the treatment of MM as initial therapy or in relapsed/ refractory cases and in transplant eligible or non-eligible patients. During the early stage of clinical use, early discontinuation of this agent often occurred owing to adverse events, such as severe peripheral neuropathy and gastrointestinal disorders. However, owing to the accumulation of clinical experience, appropriate methods of administrating this agent have been established, such as early-dose reduction, once-weekly administration [2], and the change of administration route from intravenous to subcutaneous, which enable long-term, steady treatment with BTZ.

Although most MM patients receiving continuous BTZ-containing therapy do not develop severe adverse events, the number of cases with acquired resistance to BTZ therapy has increased currently. Several clinical studies of BTZ treatment for relapsed or refractory (RR) $\mathrm{MM}$ showed that the median duration of response ranged from half-year to a year with or without dexamethasone, which supports the theory that in most of cases of continuous BTZ treatment, MM eventually acquired BTZ resistance within a year [3]. To explore effective treatment strategy for overcoming BTZ resistance, several clinical trials using new agents or combination therapy with BTZ have been performed on RR MM cases pretreated with BTZ. However, little is currently known 
about the mechanism of acquired resistance to BTZ; therefore, these trials were not always planned to target the mechanism of action of BTZ resistance in MM cases. To develop an effective treatment strategy, it is essential to address the mechanism of action of BTZ-induced anti-tumor effect, to determine how tumor cells acquire resistance to the agent, and to develop an optimal therapy targeting the specific pathway attributable to drug resistance. In this review, we summarize recent knowledge of the mechanism of BTZ-induced cell death and the factors associated with sensitivity to BTZ treatment by reviewing the previous reports on preclinical and clinical studies of MM.

\section{Role of ubiquitin-proteasome pathway in multiple myeloma cell}

The ubiquitin-proteasome system plays a crucial role in maintaining intracellular homeostasis, regulating substrates that control cell cycle, metabolism, survival, and apoptosis, and eliminating damaged proteins that are toxic to the cells. In eukaryotic cell, substrates to be degraded are conjugated by polyubiquitination via E2 and E3 ligase, subsequently leading to their degradation by $20 \mathrm{~S}$ proteasome. In general, tumor cells have a more developed proteasome pathway than normal cells [4], because tumor cells have an abundant capability for synthesis, movement, and modification of proteins, owing to their advanced cell proliferation and highly activated invasion into other organs. To compensate for the stress generated by marked synthesis and modification of proteins, tumor cells depend on the proteasome pathway, where misfolded proteins and functional proteins are uniformly degraded and remolded effectively [5]. Of the several tumor cell types, MM cells and pancreatic tumor cells, which contain many secreted proteins, are more dependent on the ubiquitin-proteasome pathway. In the case of MM cells, unfolded proteins, including monoclonal immunoglobulin and various cytokines critical for maintaining the interaction of stroma cells, easily accumulate during synthesis, adequate folding, glycosylation, and transport of newly generated proteins. To regenerate the misfolded proteins, MM cells have highly developed Endoplasmic-reticulum-associated protein degradation (ERAD) systems, by which misfolded proteins are excluded from the ER, conjugated by polyubiquitination, and subjected to proteolysis at the proteasome. Therefore, the inhibition of proteasomes would contribute to a disruption in the balance of the ERAD system, followed by an over-stress of the ER and sequential progression of apoptosis in MM cells.

\section{Endoplasmic reticulum stress and unfolded protein response in multiple myeloma cell}

BTZ is a boronic acid dipeptide that inhibits activations of $\beta 5$ and $\beta 1$ subunits of the $20 \mathrm{~S}$ proteasome core in the $26 \mathrm{~S}$ proteasome complex, and is characterized as a reversible inhibitor of the above activities [6]. Misfolded proteins and functional proteins to be degraded by the proteasome accumulate in the ER lumen and cytosol via this mechanism. This accumulation facilitates several stresses, including ER overload, the generation of excess oxygen, and a functional disorder of intracellular proteins, eventually leading to ER stress-related apoptosis $[7,8]$ and cell death due to the DNA damage $[9,10]$ (Fig. 1).

Newly synthesized proteins, secretory proteins, such as cytokines, chemokines, and immunoglobulin, are transported to the ER, folded with adequate conformation by ER chaperones, and modified as mature proteins by the addition of a complicated structure of glycoproteins at the ER and Golgi body. However, when the misfolding is too severe, the proteins are removed from the ER, added to the cytosol via polyubiquitination by E2 and E3 ligase, degraded by proteasomes, and subjected to ERAD. Therefore, the inhibition of proteasomes represses the degradation and recycling of proteins and amino acids from the misfolded proteins, causing the accumulation of these misfolded proteins in the ER [11]. The excess production of immunoglobulin and several cytokines allows MM cells to maintain contact with stroma cells in the bone marrow (BM) milieu. Therefore, MM cells can tolerate the excess ER stress, because they have a well-developed ER compared with other hematological malignant tumor cells.

In mammalian cells, unfolded protein responses (UPR) are initiated by the activation of three ER transmembrane proteins; activating transcription factor (ATF6), inositol requiring kinase 1 (IRE1 $\alpha)$ and PKR-like ER kinase (PERK) [12]. The activation of these stress sensors resulted in transcriptional up-regulation of various UPR target genes involved in ER chaperons, folding enzymes, ERAD regulator, and apoptosis initiator [13]. The up-regulation of these genes coordinates UPR as follows: (1) translational attenuation to limit further accumulation of unfolded proteins; (2) transcriptional activation of genes encoding ERresident chaperons; and (3) ER-associated degradation (ERAD) pathway to restore the folding capacity. If the cells are exposed to prolonged or strong ER stress, the cells are destroyed by apoptosis.

When proteasome action is inhibited by the bortezomib treatment, MM cells are easily exposed to uncompensated ER stress [11], which facilitates the progression of cell death rather than amelioration of cell stress by restoring damaged proteins. UPR changes its function from acting to promote 


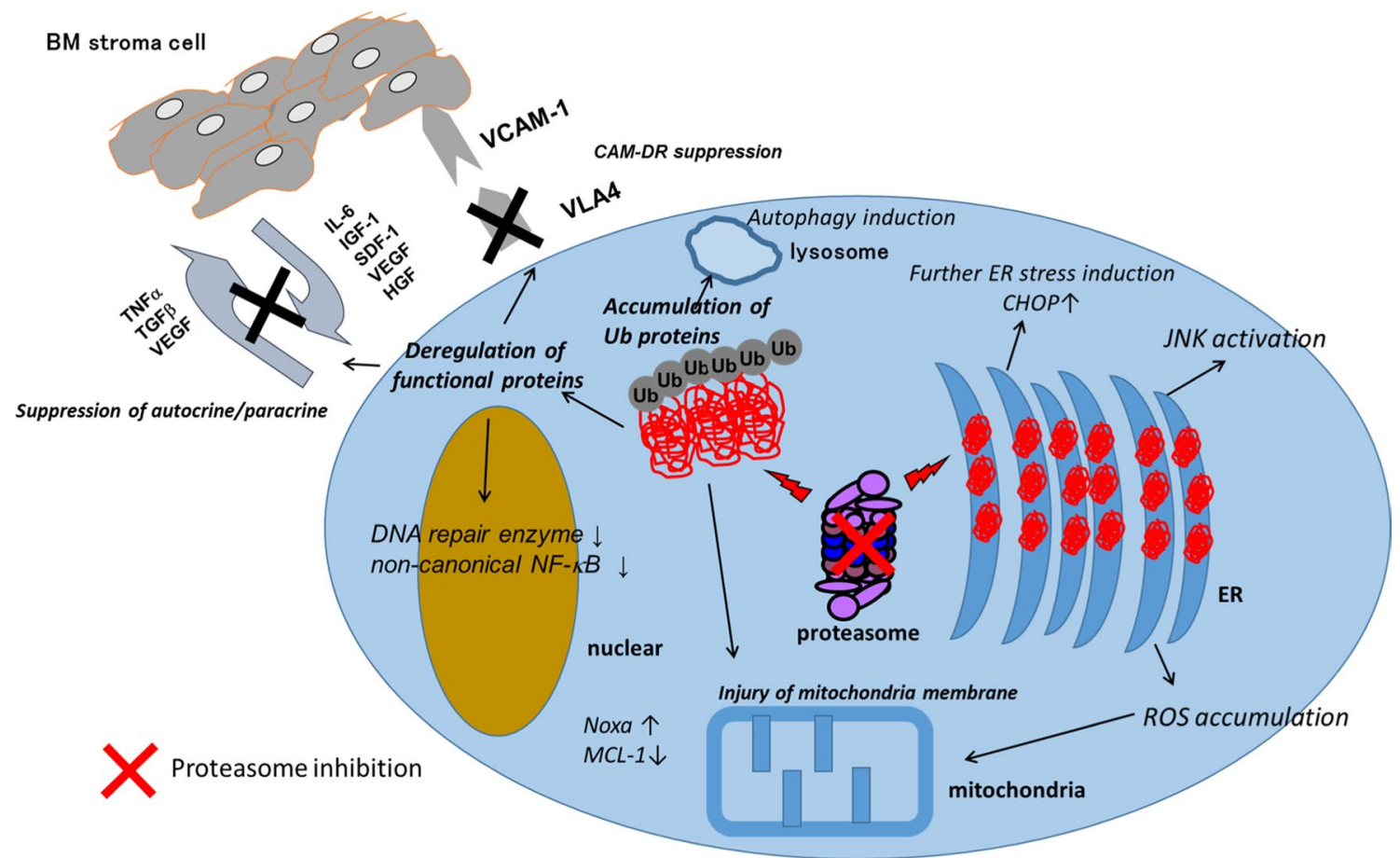

Fig. 1 Effects of proteasome inhibition on multiple myeloma cells. The inhibition of proteasome action by bortezomib (BTZ) treatment results in the accumulation of misfolded proteins and functional proteins to be degraded by the proteasome in the endoplasmic reticulum (ER) lumen and cytosol, which facilitates several stresses, including ER overload, generation of excess oxygen, and functional disorder of intracellular proteins, eventually leading to ER stress-related apopto- sis and cell death due to DNA damage. BTZ also disrupts the paracrine growth of multiple myeloma (MM) cells in the bone marrow (BM) milieu by inhibiting MM cell interaction with BM stroma cells (BMSC) through the suppression of cell adhesion molecules, such as VLA-4, in the MM cells and the secretion of IL-6 and IGF-1 in the BMSC cells cellular survival to committing the cell to apoptosis through up-regulation of pro-apoptotic factors, such as the elevation in the levels of C/EBP-homologous protein (CHOP), activation of two caspase cascades, caspase 4 and 12, DR5(TRAIL-R2) expression, and phosphorylation of c-Jun $\mathrm{N}$-terminal protein kinases, which in turn advance the caspase-dependent apoptosis of MM cells [11, 14] (Figs. 1, 2).

In the process of remodeling proteins, Reactive Oxygen Species (ROS) have played a critical role in the adequate folding of the protein complex [15]. However, by proteasome inhibition, unfolded proteins accumulate in the ER, which promotes the overproduction of ROS in the ER, resulting in fatal cell stress. Toxicity from overproduction of ROS is also known as a main downstream result of ER stress caused by proteasome inhibition and is known as a main mechanism of BTZ-induced apoptosis of MM cells [16-18].

\section{Alteration of NF- $\kappa$ B pathway by the bortezomib treatment}

The mechanism of action expected in the clinical use of BTZ is an inhibitory effect on NF- $\mathrm{B}$ activity in MM cells.
$\mathrm{NF}-\kappa \mathrm{B}$ is a complex with a dimer of different Rel families, including RelA (p65), RelB, c-Rel, p50 (NF-кB1), and p52 (NF-кB2), and has an important role in the biology of several tumors. NF- $\kappa \mathrm{B}$ activity is composed of two different pathways, canonical and non-canonical. In the canonical pathway, a heterodimer of RelA and p50 translocates into the nucleus and upregulates several survival genes (usually regulated by $\mathrm{I} \kappa \mathrm{B} \alpha$ family proteins) through transcription. Following several stimulations by various cytokines, such as IL-6, IGF-1, and TNF- $\alpha$, the I $\mathrm{B} \alpha$ protein is phosphorylated by IкB kinase (IKK) and subsequently polyubiquitinated and degraded by the $26 \mathrm{~S}$ proteasome, which promotes the translocation of RelA and the p50 complex. When the inhibition of proteasome occurs owing to BTZ administration, phosphorylated I $\mathrm{B}$ accumulates in the cytosol and prevents the translocation of RelA and the p50 complex, thereby suppressing canonical NF- $\kappa \mathrm{B}$ activity. However, unlike earlier reported studies, recent study shows that BTZ activates constitutive NF- $\kappa$ B activity in various tumor cell lines [19], suggesting the need for precise re-evaluation of BTZ activity on NF- $\kappa B$ activity in MM cells. Recently, Hideshima et al. have shown that BTZ triggered an expected increase in the expression levels of 


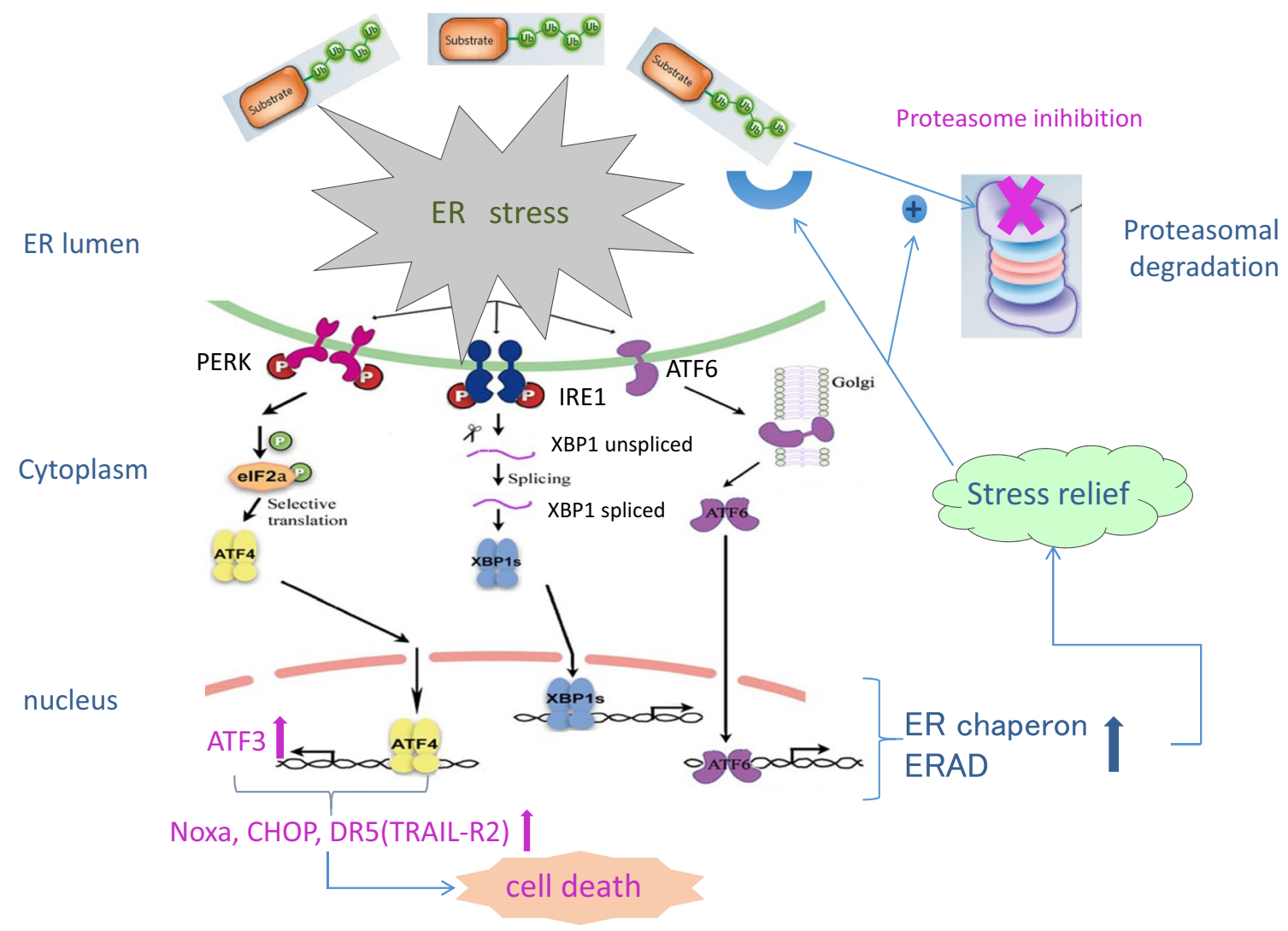

Fig. 2 Endoplasmic reticulum stress and unfolded protein response in multiple myeloma cells. Multiple myeloma cells can tolerate the excess ER stress, because unfolded protein response (UPR), including three pathways, PERK-eIF2alfa-ATF4, IRE1-XBP1, and ATF6 axis, contributes to avoid fatal ER stress through the activation of ER chaperon and the ER-associated degradation (ERAD). When fatal ER stress is triggered by the proteasome inhibition, UPR promotes the cell death via the activation of several pro-apoptotic factors, ATF families, Noxa, CHOP, and DR5 phosphorylated $\mathrm{I} \kappa \mathrm{B} \alpha$ and an unexpected downregulation of I $\mathrm{B} \alpha$, followed by constitutive NF- $\kappa \mathrm{B}$ activation mediated by nuclear translocation of the p65 complex in MM cell lines [19]. Although BTZ activates constitutive NF-кB activity, it is also possible that BTZ blocks non-canonical $\mathrm{NF}-\kappa \mathrm{B}$ activity, because the inhibition of the proteasomedependent conversion of p100-p53 is observed in BTZtreated MM cell lines. Non-canonical NF- $\mathrm{B}$ activation is also involved in the main pathogenesis of MM cells. A previous report demonstrated that the non-canonical $\mathrm{NF}-\kappa \mathrm{B}$ activity was markedly elevated in primary MM cells derived from patients [20], and the elevation of this activity was confirmed in BTZ-resistant primary MM cells from patients. Therefore, it should be considered that the alteration of NF- $\mathrm{BB}$ activity by BTZ depends on the cell type and dominant type of NF- $\kappa \mathrm{B}$ activity, canonical or non-canonical, in MM cells.

\section{Inhibition of the interaction of MM cells with bone marrow stroma cells by the bortezomib treatment}

With the interaction of bone marrow stroma cells (BMSC) via cell-to-cell contact or cytokine release, MM cells usually acquire survival benefits from BMSC cells, such as cell proliferation and resistance to therapeutic agents, socalled cell adhesion-mediated drug resistance (CAM-DR) (Fig. 1). BTZ treatment inhibited the expression of IL-6 receptor in MM cells [21], which decreased the activation of IL-6-induced ERK, STAT3, and Akt pathways, and non-canonical NF-кB activity. With BTZ treatment, MM cells lost their expression of the IL-6 receptor [21], which resulted in non-canonical NF- $\kappa \mathrm{B}$ activity and in the decreased activation of the IL-6-induced ERK, STAT3, and Akt pathways. BTZ treatment decreased the expression of several cell adhesion molecules, such as VLA-4 [22], on 
the MM cell surface, disrupted the paracrine growth of MM cells in the BM milieu, and decreased the secretion of IL-6 and IGF-1 in BMSC cells, resulting in the suppression of the MM cell's adherence to BMSC, thereby enhancing MM cell susceptibility to therapeutic agents.

\section{What factors associate with the sensitivity and resistance of bortezomib treatment?}

Several studies have tried to determine the factors associated with BTZ sensitivity in MM cells to establish new clinical biomarkers, which will contribute to identifying patients who will benefit maximally from BTZ-containing therapy, or who will experience severe adverse effects, such as peripheral neuropathy and interstitial pneumonia when BTZ is administrated. In the APEX study [23], Mannava et al. have reported that the expression level of Kruppel-like factor 9 (KLF9) is involved in the response of BTZ-containing therapy for RR MM. In this study, most patients with MM who showed a high level of KLF9 expression in primary MM cells showed good response to BTZ treatment compared to patients with low KLF9 expression. KLF9 is upregulated by Histone Deacetylase inhibition induced by BTZ treatment, and is associated with the transcriptional factor attributable to Noxa gene expression; Noxa is a BH3-only protein that serves as a pro-apoptotic factor. In other reports, the analysis of plasma cells of patients with MM collected in the APEX study showed that a high expression level of nicotinamide phosphoribosyltransferase (Nampt) is associated with a poor outcome of BTZ treatment, poor response to the treatment, and short duration of survival after the treatment [24]. Silencing of Nampt by the shRNA or HDAC inhibitor FK228, which suppresses Nampt expression by decreasing intracellular NAD + levels in MM cell lines, increased the sensitivity of MM cells to BTZ treatment compared to control cells. Using siRNA screening analysis, Zhu et al. investigated the specific factor that influences the sensitivity to BTZ treatment, and identified CDK5, cyclin-dependent kinase 5 [25]. Since CDK5 upregulates the expression of the PSMB5 gene at a transcriptional level, MM cell lines with a high expression of CDK5 showed low sensitivity to BTZ treatment compared to cell lines with low expression. Furthermore, two reagents, Roscovitine and SCH727965, which have inhibitory effects on CDK5, enhanced BTZinduced apoptosis in MM cell lines. Recently, miR-27a-5p, suppressing the expression of CDK5 by inhibiting the translation of CDK5 and mRNA [26], has been reported to be downregulated during BTZ treatment, which result in the activation of CDK5 following the up-regulation of PSMB5, considered as the compensatory effect of stress in MM cells; therefore, CDK5 inhibition enhances BTZ activity and has potential as a therapeutic option.
Proteasome inhibition causes the accumulation of misfolded proteins; hence, it initiates ER stress followed by an unfolded protein response (UPR), which has crucial roles in the cytotoxic effect of PI. Thus, the magnitude of dependence on the proteasome and the UPR may affect MM cells' sensitivity to PI. Ling et al. and Gambella et al. have reported that XBP1, a downstream of activated IRE1 involved in UPR, is associated with the clinical outcome of MM patients who received BTZ-containing therapy. In their reports, a low expression of the XBP1 gene is observed in primary MM samples from patients who respond poorly to bortezomib and dexamethasone (BD) treatment [27], and a high expression of XBP1 is defined as a good prognosis marker of survival among patients receiving BTZ-containing regimen [28]. In our study, we investigated 57 patients with RR MM treated with BD in Nagoya City University Hospital (Fig. 3a, b). The expression levels of proteasome- and ER stressrelated genes in primary tumor samples obtained prior to BD treatment were evaluated in association with clinical outcomes. Among those, a couple of genes encoding for the ATF family seemed to correlate with sensitivity to BTZ. We identified that the expression level of two ATF genes, ATF3 and ATF4, was lower in the shorter PFS group (PFS $<6$ months) than in the longer PFS group (Fig. 3a) [29]. In addition, cut-off values of the ATF3 and ATF4 expression level adjusted by the $\beta$-actin expression level were defined as 1.0 and 0.135 , respectively, which showed that MM patients with high expression levels of both ATF genes showed longer PFS than the other group harboring low expression level of either gene, (median PFS: 9.0 vs 3.2 months) (Fig. 3b). We speculate that MM cells with a high expression of ATF genes pose the conventional ER stress, thus proteasome inhibition by BTZ exposure could trigger further ER overloads followed by an uncompensated ER cell stress even with UPR activation and rapid apoptosis progression in these cells. The previous reports and our recent study showed that the suppression of ATF3 and ATF4 by an RNA-interfering method has inhibited death receptor DR5 expression and the upregulation of Noxa, a pro-apoptotic BH3-only protein, by BTZ treatment in MM cells. Using chip analysis from MM cell lines and primary MM samples from patients, we also confirmed that the complex of ATF3 and ATF4 works as a transcriptional factor of the Noxa gene. In summary, from the analysis of primary tumor samples from patients received BTZ-containing therapy, KLF9, Nampt, CDK5, and three ER stress- and UPR- associated markers (XBP1, ATF3, and AFT4) were identified as a predictive markers of sensitivity of BTZ treatment. However, these factors have not been fully evaluated, and it seems to be difficult to clearly predict the efficacy of BTZ treatment using a single factor, owing to the heterogeneity and complexity 

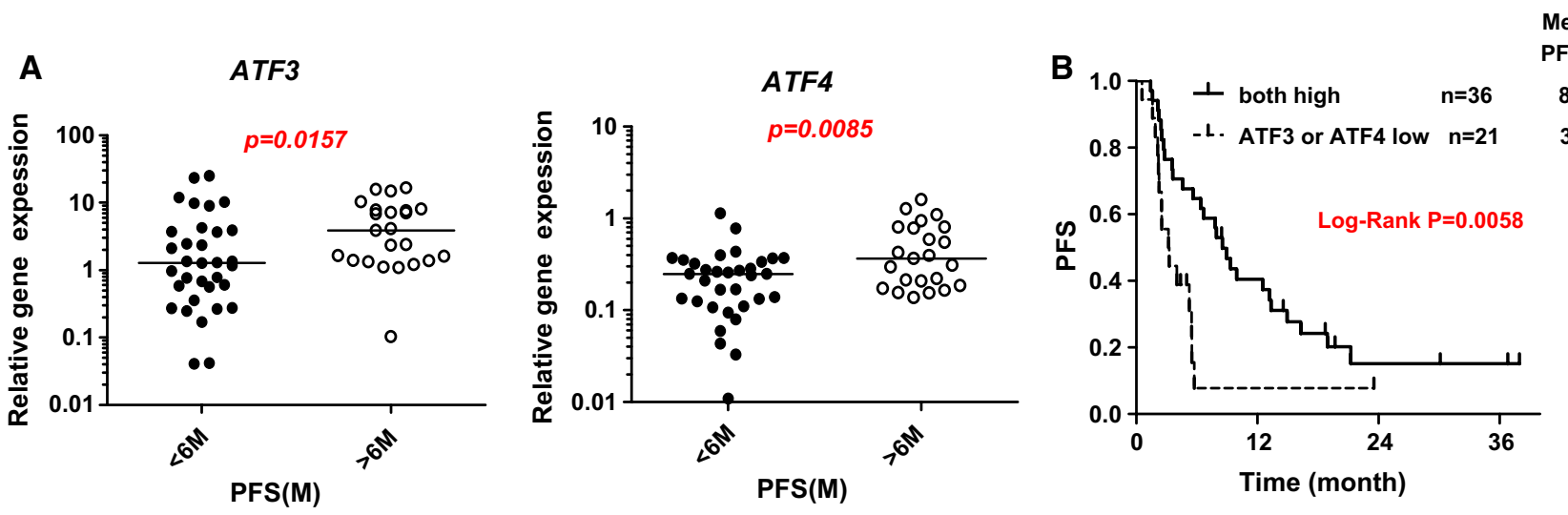

Fig. 3 Two ATF genes are involved in the clinical outcome of patients with multiple myeloma receiving the bortezomib plus dexamethasone therapy. a Higher expression levels of ATF3 and ATF4 correlate with longer duration of response in 57 patients with relapsed/refractory multiple myeloma (MM) treated with bortezomib and dexamethasone in Nagoya City University Hospital. Each bar represents the median value of progression-free survival (PFS). Comparison of gene expression between two groups with longer and shorter PFS after bortezomib plus dexamethasone therapy was made using the Mann-Whitney $U$ test. In this study, $P<0.05$ was considered significant. b Higher expression levels of both ATF3 and ATF4 are associated with longer PFS in patients with relapsed/refractory MM treated with bortezomib plus dexamethasone. Survival analysis used the Kaplan-Meier estimate. In this study, $P<0.05$ was considered significant
A
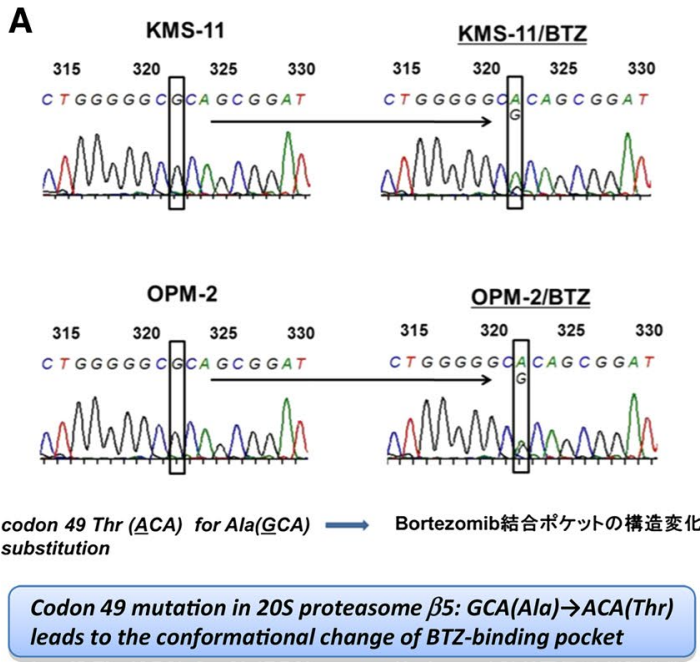

Fig. 4 BTZ-resistant MM cell lines: a role for mutated PSMB5 in preventing the accumulation of unfolded proteins and fatal ER stress. a, b Bortezomib (BMZ)-resistant multiple myeloma (MM) cell lines harbored exactly the same mutation at codon 49 of PSMB5, which
B

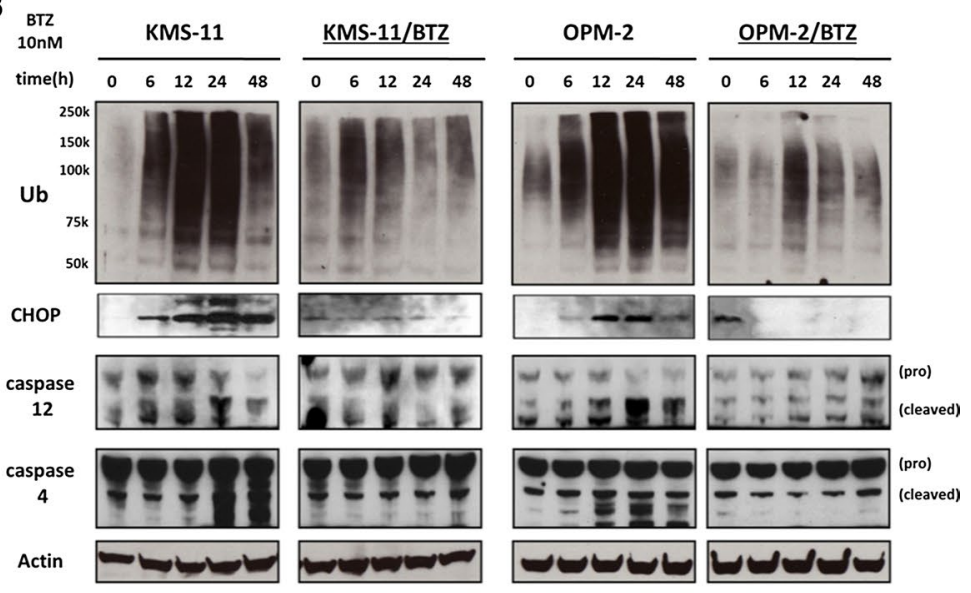

of bioactivity in MM cells and the mechanism of action of BTZ-induced cell death.

So far, several studies have reported the mechanism of action of BTZ resistance using the artificially established MM cell lines with resistance to the action of BTZ. Among them, we have previously reported that the mutation of PSMB5 is involved in BTZ resistance in MM cells (Fig. 4a, b) [30], and others have reported several factors upregulated in BTZ-resistant MM cell lines, including IGF-1R leads to the conformational change of the BTZ-binding pocket. Even in the presence of BTZ, polyubiquitinated protein does not accumulate, and apoptosis initiators, such as CHOP, caspase-12, caspase-4, and Noxa, are not activated

[31], PSMB2 [32, 33], germinal center B cell markers [34], and TXNRD2 [16]. Another reported reduced expression of the plasma cell differentiation marker $[35,36]$ or XBP1 $[37,38]$ in BTZ-resistant cells. In their reports, Chungyee et al. $[37,38]$ have proposed that the BTZ resistant clone would be generated from the population with a low production of monoclonal immunoglobulin and less ER stress, such as pre-plasmablast population, which is capable to survive and growth during the inhibition of proteasome 


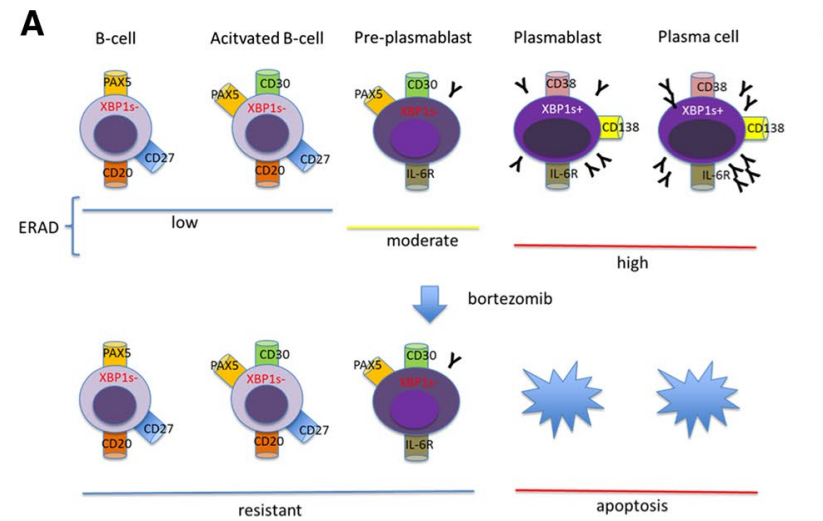

Fig. 5 Model of proteasome inhibitor resistance associated with low level of protein synthesis and expression of XBP1. a Multiple myeloma may have several neoplastic subpopulations, including B cells, activated B cells, pre-plasma blasts, plasma blasts, and plasma cells. Among them, B cells, activated B cells, and pre-plasma blasts have low levels of ER-associated protein degradation (ERAD) and spliced $\mathrm{X}$-box binding protein 1 (sXBP-1), and they are low sensitive to the

(Fig. 5). Further investigation using primary MM samples and next-generation sequencing analysis is necessary to find predictive biomarkers for BTZ resistance and to develop effective treatment strategies for overcoming BTZresistant MM.

\section{Conclusion}

By the accumulation of studies of BTZ, the mechanism of action of the BTZ-induced effect on MM cells has emerged gradually, with a better understanding of not only NF-KB signaling but also ER stress and subsequently the URR system in MM cells. In addition, several factors associated with BTZ sensitivity have also been identified, which contribute to understanding the differences in the biological activity of MM cells with respect to their dependence on proteasome activity and UPR. Further study of the mechanism of BTZinduced activity is needed to establish a well-characterized predictive marker of BTZ response and to develop a potent treatment strategy for this incurable disease.

\section{References}

1. Ogawa Y, Tobinai K, Ogura M, Ando K, Tsuchiya T, Kobayashi $\mathrm{Y}$, et al. Phase I and II pharmacokinetic and pharmacodynamic study of the proteasome inhibitor bortezomib in Japanese patients with relapsed or refractory multiple myeloma. Cancer Sci. 2008;99:140-4.

2. Tokuhira M, Watanabe R, Nemoto T, Hanzawa K, Sagawa M, Tomikawa T, et al. Successful treatment with a modified bortezomib schedule of weekly and longer intervals for patients with refractory/resistance multiple myeloma. Leuk Res. 2011;35:591-7.
B

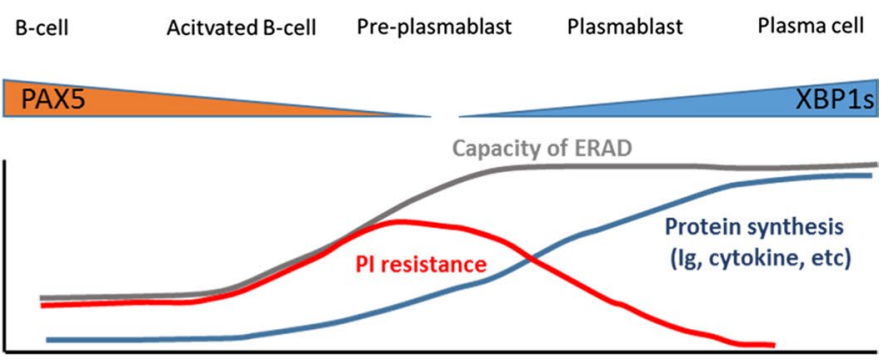

effect of proteasome inhibition. Other subpopulations, plasma blasts and plasma cells, have high abundant of immunoglobulin secretion, with the high capacity of ERAD and activated spliced XBP1. They are susceptible to the proteasome inhibition. b Schema comparing the relative ERAD capacity, protein synthesis, and susceptibility of proteasome inhibition in plasma cells and their precursors

3. Jagannath S, Barlogie B, Berenson J, Siegel D, Irwin D, Richardson PG, et al. A phase 2 study of two doses of bortezomib in relapsed or refractory myeloma. $\mathrm{Br} \mathrm{J}$ Haematol. 2004;127:165-72.

4. Adams J. The development of proteasome inhibitors as anticancer drugs. Cancer Cell. 2004;5:417-21.

5. Reimold AM, Iwakoshi NN, Manis J, Vallabhajosyula P, Szomolanyi-Tsuda E, Gravallese EM, et al. Plasma cell differentiation requires the transcription factor XBP-1. Nature. 2001;412:300-7.

6. Crawford LJ, Walker B, Ovaa H, Chauhan D, Anderson KC, Morris TC, et al. Comparative selectivity and specificity of the proteasome inhibitors BzLLLCOCHO, PS-341, and MG-132. Cancer Res. 2006;66:6379-86.

7. Chauhan D, Hideshima T, Mitsiades C, Richardson P, Anderson KC. Proteasome inhibitor therapy in multiple myeloma. Mol Cancer Ther. 2005;4:686-92.

8. Ri M, Iida S, Ishida T, Ito A, Yano H, Inagaki A, et al. Bortezomib-induced apoptosis in mature T-cell lymphoma cells partially depends on upregulation of Noxa and functional repression of Mcl-1. Cancer Sci. 2009;100:341-8.

9. Hideshima T, Mitsiades C, Akiyama M, Hayashi T, Chauhan $\mathrm{D}$, Richardson $\mathrm{P}$, et al. Molecular mechanisms mediating antimyeloma activity of proteasome inhibitor PS-341. Blood. 2003;101:1530-4.

10. Mitsiades N, Mitsiades CS, Richardson PG, Poulaki V, Tai YT, Chauhan D, et al. The proteasome inhibitor PS-341 potentiates sensitivity of multiple myeloma cells to conventional chemotherapeutic agents: therapeutic applications. Blood. 2003;101:2377-80.

11. Obeng EA, Carlson LM, Gutman DM, Harrington WJ Jr, Lee KP, Boise LH. Proteasome inhibitors induce a terminal unfolded protein response in multiple myeloma cells. Blood. 2006;107:4907-16.

12. Ron D, Walter P. Signal integration in the endoplasmic reticulum unfolded protein response. Nat Rev Mol Cell Biol. 2007;8:519-29.

13. Rao RV, Bredesen DE. Misfolded proteins, endoplasmic reticulum stress and neurodegeneration. Curr Opin Cell Biol. 2004;16:653-62.

14. Yoshida H. ER stress and diseases. FEBS J. 2007;274:630-58. 
15. Shimizu Y, Hendershot LM. Oxidative folding: cellular strategies for dealing with the resultant equimolar production of reactive oxygen species. Antioxid Redox Signal. 2009;11:2317-31.

16. Fink EE, Mannava S, Bagati A, Bianchi-Smiraglia A, Nair JR, Moparthy K, et al. Mitochondrial thioredoxin reductase regulates major cytotoxicity pathways of proteasome inhibitors in multiple myeloma cells. Leukemia. 2016;30:104-11.

17. Fribley A, Zeng Q, Wang CY. Proteasome inhibitor PS-341 induces apoptosis through induction of endoplasmic reticulum stress-reactive oxygen species in head and neck squamous cell carcinoma cells. Mol Cell Biol. 2004;24:9695-704.

18. Perez-Galan P, Roue G, Villamor N, Montserrat E, Campo $\mathrm{E}$, Colomer D. The proteasome inhibitor bortezomib induces apoptosis in mantle-cell lymphoma through generation of ROS and Noxa activation independent of p53 status. Blood. 2006; 107:257-64.

19. Hideshima T, Ikeda H, Chauhan D, Okawa Y, Raje N, Podar K, et al. Bortezomib induces canonical nuclear factor-kappaB activation in multiple myeloma cells. Blood. 2009;114:1046-52.

20. Keats JJ, Fonseca R, Chesi M, Schop R, Baker A, Chng WJ, et al. Promiscuous mutations activate the noncanonical NF-kappaB pathway in multiple myeloma. Cancer Cell. 2007;12:131-44.

21. Hideshima T, Chauhan D, Hayashi T, Akiyama M, Mitsiades N, Mitsiades C, et al. Proteasome inhibitor PS-341 abrogates IL-6 triggered signaling cascades via caspase-dependent downregulation of gp130 in multiple myeloma. Oncogene. 2003;22:8386-93.

22. Noborio-Hatano K, Kikuchi J, Takatoku M, Shimizu R, Wada T, Ueda $\mathrm{M}$, et al. Bortezomib overcomes cell-adhesion-mediated drug resistance through downregulation of VLA-4 expression in multiple myeloma. Oncogene. 2009;28:231-42.

23. Mannava S, Zhuang D, Nair JR, Bansal R, Wawrzyniak JA, Zucker SN, et al. KLF9 is a novel transcriptional regulator of bortezomib- and LBH589-induced apoptosis in multiple myeloma cells. Blood. 2012;119:1450-8.

24. Cagnetta A, Cea M, Calimeri T, Acharya C, Fulciniti M, Tai $\mathrm{YT}$, et al. Intracellular NAD $(+)$ depletion enhances bortezomibinduced anti-myeloma activity. Blood. 2013;122:1243-55.

25. Zhu YX, Tiedemann R, Shi CX, Yin H, Schmidt JE, Bruins LA, et al. RNAi screen of the druggable genome identifies modulators of proteasome inhibitor sensitivity in myeloma including CDK5. Blood. 2011;117:3847-57.

26. Ballabio E, Armesto M, Breeze CE, Manterola L, Arestin M, Tramonti D, et al. Bortezomib action in multiple myeloma: microRNA-mediated synergy (and miR-27a/CDK5 driven sensitivity)? Blood Cancer J. 2012;2:e83.

27. Ling SC, Lau EK, Al-Shabeeb A, Nikolic A, Catalano A, Iland $\mathrm{H}$, et al. Response of myeloma to the proteasome inhibitor bortezomib is correlated with the unfolded protein response regulator XBP-1. Haematologica. 2012;97:64-72.

28. Gambella M, Rocci A, Passera R, Gay F, Omede P, Crippa C, et al. High XBP1 expression is a marker of better outcome in multiple myeloma patients treated with bortezomib. Haematologica. 2014;99:e14-6.

29. Narita T, Ri M, Masaki A, Mori F, Ito A, Kusumoto S, et al. Lower expression of activating transcription factors 3 and 4 correlates with shorter progression-free survival in multiple myeloma patients receiving bortezomib plus dexamethasone therapy. Blood Cancer J. 2015;5:e373.

30. Ri M, Iida S, Nakashima T, Miyazaki H, Mori F, Ito A, et al. Bortezomib-resistant myeloma cell lines: a role for mutated PSMB5 in preventing the accumulation of unfolded proteins and fatal ER stress. Leukemia. 2010;24:1506-12.

31. Kuhn DJ, Berkova Z, Jones RJ, Woessner R, Bjorklund CC, Ma $\mathrm{W}$, et al. Targeting the insulin-like growth factor- 1 receptor to overcome bortezomib resistance in preclinical models of multiple myeloma. Blood. 2012;120:3260-70.

32. Kraus J, Kraus M, Liu N, Besse L, Bader J, Geurink PP, et al. The novel beta2-selective proteasome inhibitor LU-102 decreases phosphorylation of I kappa B and induces highly synergistic cytotoxicity in combination with ibrutinib in multiple myeloma cells. Cancer Chemother Pharmacol. 2015;76:383-96.

33. Kraus M, Bader J, Geurink PP, Weyburne ES, Mirabella AC, Silzle T, et al. The novel beta2-selective proteasome inhibitor LU-102 synergizes with bortezomib and carfilzomib to overcome proteasome inhibitor resistance of myeloma cells. Haematologica. 2015;100:1350-60.

34. Stessman HA, Mansoor A, Zhan F, Linden MA, Van Ness B, Baughn LB. Bortezomib resistance can be reversed by induced expression of plasma cell maturation markers in a mouse in vitro model of multiple myeloma. PLoS ONE. 2013;8:e77608.

35. Jung HJ, Chen Z, Fayad L, Wang M, Romaguera J, Kwak $\mathrm{LW}$, et al. Bortezomib-resistant nuclear factor kappaB expression in stem-like cells in mantle cell lymphoma. Exp Hematol. 2012;40(107-18):e2.

36. Perez-Galan P, Mora-Jensen H, Weniger MA, Shaffer AL 3rd, Rizzatti EG, Chapman CM, et al. Bortezomib resistance in mantle cell lymphoma is associated with plasmacytic differentiation. Blood. 2011;117:542-52.

37. Leung-Hagesteijn C, Erdmann N, Cheung G, Keats JJ, Stewart $\mathrm{AK}$, Reece DE, et al. Xbp1 s-negative tumor B cells and preplasmablasts mediate therapeutic proteasome inhibitor resistance in multiple myeloma. Cancer Cell. 2013;24:289-304.

38. Orlowski RZ. Why proteasome inhibitors cannot ERADicate multiple myeloma. Cancer Cell. 2013;24:275-7. 